\title{
MODELLING JOINT AUTOREGRESSIVE MOVING AVERAGE PROCESSES
}

\author{
ROSS S. BOWDEN
}

(Received 31 May 2018; first published online 1 August 2018)

2010 Mathematics subject classification: primary 62M10; secondary 60G10.

Keywords and phrases: time series, replicated, hypothesis tests, estimation.

This thesis defines, identifies, fits and applies models for replicated stationary and invertible time series processes which are represented by so-called joint ARMA (autoregressive moving average) or JARMA processes. These are processes that have shared ARMA coefficients with innovations variances that are in common (replicated ARMA or RARMA processes), vary with the series (almost identical ARMA or AIARMA processes) or are proportional to the series mean (conditional AIARMA or CAIARMA processes). For a shared innovations variance matrix, the extension to joint VARMA (replicated vector ARMA) processes is undertaken. All models include extraneous variables.

It is proven that a RARMA process can be represented by one stationary and invertible univariate series using interleaving and this is shown to also apply to replicated VARMA processes. The interleaving of the replicated series allows ARMA and VARMA models to be fitted to all series simultaneously using readily available time series software. The spectral density of an interleaved replicated ARMA (and VARMA) process is shown to be a compressed version of the shared spectral density of each of the replicated series.

In order to identify JARMA processes, tests for comparing time series were investigated. The system of testing for process equivalence begins with testing for the same spectral shape. If accepting of the same shape, testing is undertaken for the same scale.

To improve on the power of the existing tests and/or develop tests that are simpler to implement, seven new tests are proposed. These tests include four new shape tests

Thesis submitted to Murdoch University in August 2017; degree awarded on 7 December 2017; supervisors Brenton Clarke and Nicola Armstrong.

(C) 2018 Australian Mathematical Publishing Association Inc. 
using differences across frequencies in the logged ratio of the periodogram ordinates, the distance between the sample autocorrelation functions and the calculation of the sample variance of the logged ratios. The three new scale tests employ the Wald test, the mean logged ratios and the Central Limit Theorem.

Size and power simulation studies of the new and existing tests indicate that the sizes for all tests are slightly higher than their nominal values. The most powerful shape tests are the autocorrelation test, the $(N / 2)$ th difference test and the existing likelihood ratio regression test. Of the scale tests, the mean logged ratio and the Central Limit Theorem tests show comparative power with the existing likelihood ratio test.

A graphical procedure was developed to explore whether two series have the same spectral shape and scale and to determine their common ARMA order. The method uses the shape test [3], the Central Limit Theorem scale test and a merging of the autocorrelation functions.

Unconditional maximum likelihood estimates of RARMA, AIARMA and CAIARMA models were derived analytically and numerically using unconstrained and constrained joint likelihood and interleaving approaches. In the simulation estimation studies, these three methods all use the $\mathrm{R}$ function, arima, as part of the optimisation process with some adjustment to the inputs and outputs. This avoids the preparation of extensive bespoke coding.

The asymptotic distributions of the maximum likelihood estimates were analytically derived and show a natural progression from the known results for single-series ARMA models. Extensive simulation studies were conducted into the properties of the maximum likelihood estimates of the ARMA, RARMA, AIARMA and CAIARMA model parameters.

Across most models, the estimates have low bias even for small samples, show accurate coverage for the associated confidence intervals and follow the asymptotic standard errors. Hence, the asymptotic distribution of the estimates can be used as a reliable indicator of estimate behaviour. Moreover, the Hessian-based empirical confidence intervals appear to be reliable. The estimates do not perform well when the ARMA coefficients are near the unit circle nor when the AR and MA polynomials in the backshift operator are close to cancelling.

An area of some interest in the literature is the contrast in estimator efficiency between using a small number of long series or a large number of short series. For RARMA, AIARMA and CAIARMA versus single-series ARMA processes, the asymptotic and simulation results as well as the interleaving method indicate that it is the total length of all series that is the determining factor when estimating common parameters.

The interleaving method for both univariate and multivariate time series was applied to over sixty years of daily maximum and minimum temperatures for Perth, Western Australia [1, 2]. This shows that there have been changes in mean temperatures due to location and climate change. The location change estimates suggest that the current recording site, $5 \mathrm{~km}$ north of Perth, is $1.8^{\circ} \mathrm{C}$ colder overnight than the original site at King's Park near the city. 


\section{References}

[1] R. S. Bowden and B. R. Clarke, 'A single series representation of multiple independent ARMA processes', J. Time Series Anal. 33 (2012), 304-311.

[2] R. S. Bowden and B. R. Clarke, 'Using multivariate time series to estimate location and climate change effects on temperatures employed in future electricity demand simulation', Aust. N. Z. J. Stat. 59 (2017), 413-431.

[3] D. S. Coates and P. J. Diggle, 'Tests for comparing two estimated spectral densities', J. Time Series Anal. 7 (1986), 7-20.

ROSS S. BOWDEN, Mathematics and Statistics,

School of Engineering and Information Technology,

Murdoch University, Murdoch WA 6150, Australia

e-mail: ross.bowden@iinet.net.au 